\title{
Bulk Heating Effects as Tests for Collapse Models
}

\author{
Stephen L. Adler* \\ Institute for Advanced Study, Einstein Drive, Princeton, NJ 08540, USA.
}

\author{
Andrea Vinante ${ }^{\dagger}$ \\ Department of Physics and Astronomy, University of Southampton, SQ17 1BJ, United Kingdom
}

We discuss limits on the noise strength parameter in mass-proportional-coupled wave function collapse models implied by bulk heating effects, and examine the role of the noise power spectrum in comparing experiments of different types. This comparison utilizes a calculation of the rate of heating through phonon excitation implied by a general noise power spectrum $\lambda(\omega)$. We find that in the standard heating formula, the reduction rate $\lambda$ is replaced by $\lambda_{\text {eff }}=\frac{2}{3 \pi^{3 / 2}} \int d^{3} w e^{-\vec{w}^{2}} \vec{w}^{2} \lambda\left(\omega_{L}\left(\vec{w} / r_{c}\right)\right)$, with $\omega_{L}(\vec{q})$ the longitudinal acoustic phonon frequency as a function of wave number $\vec{q}$, and with $r_{C}$ the noise correlation length. Hence if the noise power spectrum is cut off below $\omega_{L}\left(|\vec{q}| \sim r_{c}^{-1}\right)$, the bulk heating rate is sharply reduced, allowing compatibility of current experimental results. We suggest possible new bulk heating experiments that can be performed subject to limits placed by natural heating from radioactivity and cosmic rays. The proposed experiments exploit the vanishing of thermal transport in the low temperature limit.

\section{INTRODUCTION AND COMPARISON OF BOUNDS ON THE EFFECTIVE NOISE COUPLING}

There is increasing interest in testing wave function collapse models [1], and in particular the continuous spontaneous localization (CSL) model, by searching for effects associated with the small noise which drives wave function collapse when nonlinearly coupled in the Schrödinger equation. The original proposals for the noise coupling strength were so small that devising suitable experiments was problematic, but the situation has changed with the suggestion [2] that latent image formation, such as deposition of a developable track in an emulsion or in an etched track detector, already constitutes a measurement embodying wave function collapse and so requires an enhanced noise coupling. A recent cantilever experiment of Vinante et al. [3] has set bounds consistent with the parameters suggested in [2], and reports a possible noise signal. Thus, it is timely to consider

\footnotetext{
*Electronic address: adler@ias.edu

${ }^{\dagger}$ Electronic address: A.Vinante@soton.ac.uk
} 
further experiments [4] which could detect or rule out a noise coupling with the strength suggested by [3].

For a body comprised of a group of particles of total mass $M$, the center-of-mass energy gain for white noise with mass-proportional coupling is given by the standard formula [5]

$$
\frac{d E}{d t}=\frac{3}{4} \lambda \frac{\hbar^{2}}{r_{C}^{2}} \frac{M}{m_{N}^{2}}
$$

with $m_{N}$ the nucleon mass and $\lambda$ the coupling parameter for white noise with noise correlation length $r_{C}$ and no frequency spectrum cutoff. Dividing by $M$, Eq. (1) can be rewritten as a formula for the energy gain rate per unit mass,

$$
\frac{d E}{d t d M}=\frac{3}{4} \lambda \frac{\hbar^{2}}{r_{C}^{2}} \frac{1}{m_{N}^{2}}
$$

For the noise coupling parameter $\lambda=10^{-7.7} \mathrm{~s}^{-1}$ suggested in [3], and the conventional value $r_{C}=10^{-5} \mathrm{~cm}$, Eq. (2) corresponds to

$$
\frac{d E}{d t d M} \simeq 40 \frac{\mathrm{MeV}}{\mathrm{g} \mathrm{s}} \simeq 0.64 \times 10^{-8} \frac{\mathrm{W}}{\mathrm{kg}} .
$$

Since heating rates of $100 \mathrm{pW} / \mathrm{kg}=10^{-10} \mathrm{~W} / \mathrm{kg}$ are attained in low temperature experiments [6], with the limit accounted for by modeling energy deposition from radioactive decays and penetrating muons [7], the residual heating from unknown sources is limited to roughly $10^{-11} \mathrm{~W} / \mathrm{kg}$. This means that the effective $\lambda$ for bulk heating is at most $3.1 \times 10^{-11} \mathrm{~s}^{-1}$, ruling out a collapse model white noise interpretation of the excess noise reported in [3].

A similar bound on the bulk heating rate is given by Earth's energy balance. Table 6.3 of de Pater and Lissauer [8] gives the luminosity to mass ratio for solar system objects, with a value $6.4 \times 10^{-12} \mathrm{~W} / \mathrm{kg}$ for Earth. Estimates of primordial and radiogenic sources of Earth heat roughly account for this, but have uncertainties that could allow $\sim 3 \times 10^{-12} \mathrm{~W} / \mathrm{kg}$ to come from unknown heating sources. This places a limit of $\sim 10^{-11} \mathrm{~s}^{-1}$ on the effective $\lambda$ for bulk heating. There is a caveat here, because as noted in [2], when the effects of dissipation are included, as in the model of Bassi, Ippoliti, and Vacchini [9], the rate of heat production can vanish at large times where a limiting temperature is reached. For example, with the parameters of [9], a limiting temperature of $0.1 \mathrm{~K}$ is reached on a time scale of billions of years, giving a current noise-induced Earth heat production rate smaller than given by Eq. (2), permitting a larger effective $\lambda$.

The effective CSL model $\lambda$ for bulk heating experiments can be strongly reduced if the noise is non-white, with a power spectrum cutoff. A spectral energy cutoff is already suggested by experimental limits on spontaneous gamma ray emission from germanium [10], which shows that 
the noise strength suggested in [2] is ruled out unless the noise power spectrum cuts off at an angular frequency below $\sim 15 \mathrm{keV} / \hbar \sim 2 \times 10^{19} \mathrm{~s}^{-1}$. For bulk heating of solids the noise couples through longitudinal acoustic phonon excitation, and with $r_{c} \sim 10^{-5} \mathrm{~cm}$, heating takes place only if the noise has frequency components of at least $\omega_{L}\left(|\vec{q}|=r_{c}^{-1}\right) \sim v_{s}|\vec{q}| \sim 0.4 \times 10^{11} \mathrm{~s}^{-1}$, with $\omega_{L}(\vec{q})$ the longitudinal phonon frequency at wave number $\vec{q}$, and $v_{s}$ the speed of sound (which for this estimate we have taken as $4000 \mathrm{~m} / \mathrm{s}$ characteristic of copper at low temperature). A quantitative calculation given in the Appendix shows that for non-white noise with power spectrum $\lambda(\omega)$ heating a solid by phonon excitation, Eq. (2) is replaced by

$$
\frac{d E}{d t d M}=\frac{3}{4} \lambda_{\mathrm{eff}} \frac{\hbar^{2}}{r_{C}^{2}} \frac{1}{m_{N}^{2}}
$$

with $\lambda_{\text {eff }}$ given by

$$
\lambda_{\text {eff }}=\frac{2}{3 \pi^{3 / 2}} \int d^{3} w e^{-\vec{w}^{2}} \vec{w}^{2} \lambda\left(\omega_{L}\left(\vec{w} / r_{c}\right)\right)
$$

When $\lambda(\omega)$ is a constant independent of $\omega$, Eq. (5) reduces to $\lambda_{\text {eff }}=\lambda$, and Eq. (2) is recovered, but when there is a frequency cutoff below the phonon excitation frequency, the effective noise coupling is strongly reduced. Thus, if the noise reported in [3] at the very low cantilever frequency of $8174 \mathrm{~s}^{-1}$ were due to CSL, it would be further evidence for non-white CSL model noise.

\section{ALTERNATIVE EXPERIMENTS FOR BOUNDING $\lambda_{\text {eff }}$}

Assuming now a maximum value $\lambda_{\text {eff }} \sim 10^{-11} \mathrm{~s}^{-1}$ consistent with low temperature experiments and non-dissipative Earth heating, let us explore possible alternative experiments for detecting or further bounding $\lambda_{\text {eff }}$. Feasibility of such experiments assumes (a) that the background heating rate from cosmic ray muons and radioactive decays can be reduced much below $3 \times 10^{-12} \mathrm{~W} / \mathrm{kg}$ by shielding, underground operation, and careful choice of materials, and (b) all other known sources of heat leaks in ultralow temperature experiments, such as vibrations, relaxation from two-level systems, and hydrogen ortho-para conversion, have been suppressed. To present estimates we multiply $3 \times 10^{-12} \mathrm{~W} / \mathrm{kg}$ by the solid density $\rho$, so that the maximum allowed value of $\lambda_{\text {eff }}$ corresponds to a volume heating rate of

$$
H=3 \times 10^{-15} \rho \mathrm{W} / \mathrm{cm}^{3},
$$

with $\rho$ the density in units $\mathrm{g} / \mathrm{cm}^{3}$. We consider two geometries for which the heat transport problem is effectively one dimensional and easily solved. 
1. For a sphere of radius $R \mathrm{~cm}$ and density $\rho$, the total heating rate is $4 \pi R^{3} H / 3$, and so the steady state rate of escape of heat from unit area of the surface will be

$$
\dot{Q}_{\text {sphere }}=R H / 3
$$

in units $\mathrm{W} / \mathrm{cm}^{2}$. This must balance the rate of transport of heat per unit area from the surface of the sphere, at temperature $T_{1}$, to the surrounding cryostat surfaces, at temperature $T_{2}$. This is given [6] in units $\mathrm{W} / \mathrm{cm}^{2}$ by the formula

$$
\dot{Q}_{\text {transport }}=5.67 \times 10^{-12} \epsilon\left(T_{1}^{4}-T_{2}^{4}\right)[K]^{4}+0.02 a P[\operatorname{mbar}]\left(T_{1}-T_{2}\right)[K],
$$

with the first term the Stefan-Boltzmann equation for radiative heat transfer, and the second term, which is linear in the pressure $P$, coming from gas particle conduction in the cryostat. Here $a \leq 1$ is an "accommodation coefficient" for gas particles on the cryostat walls, which can be as small as 0.02 for a clean metal surface in contact with helium gas, and $\epsilon \leq 1$ is the emissivity for radiative transfer. The steady state surface temperature of the sphere $T_{1}$ is determined by equating $\dot{Q}_{\text {sphere }}$ to $\dot{Q}_{\text {transport }}$. Taking as an example the density of lead $\rho=11.4 \mathrm{~g} / \mathrm{cm}^{3}$, and a sphere of radius $R=50 \mathrm{~cm}$ (which would fit in the CUORE underground experiment cryostat [11]), we have

$$
\dot{Q}_{\text {sphere }}=5.7 \times 10^{-13},
$$

while taking $a=0.02$ and $P=10^{-6}$ mbar gives

$$
\dot{Q}_{\text {transport }}=5.67 \times 10^{-24} \epsilon\left(T_{1}^{4}-T_{2}^{4}\right)[\mathrm{mK}]^{4}+4 \times 10^{-13}\left(T_{1}-T_{2}\right)[\mathrm{mK}] \quad,
$$

both in units $\mathrm{W} / \mathrm{cm}^{2}$. Evidently, for millikelvin $T_{1}$ and $T_{2}$ radiative heat transfer is completely negligible and Eqs. (9) and (10) are of similar size at the relatively high pressure of $10^{-6}$ mbar. In real millikelvin cryostats the actual residual pressure is typically orders of magnitude lower than $10^{-6}$ mbar because the vapor pressure of anything, including helium, drops to zero exponentially with decreasing temperature. The heating rate $\mathrm{H}$ can therefore be estimated by measuring the steady state surface temperature as a function of the gas pressure. Fast and sensitive measurements of temperature in the range of a few $\mathrm{mK}$ can be readily done using resistive probes. For high accuracy, SQUID-based noise thermometry has been recently demonstrated to be very effective down to $42 \mu \mathrm{K}$ [12].

Similar reasoning gives the temperature distribution inside a sphere of material with thermal conductivity $k(T)$. At a given distance $r$ from the center of the sphere, the energy transport 
rate through the spherical surface of radius $r$ is equal to

$$
E_{\text {out }}=-4 \pi r^{2} k(T) \frac{d T}{d r}
$$

which in steady state must balance the heating rate of the volume within radius $r$,

$$
E_{\text {in }}=\frac{4 \pi}{3} r^{3} H
$$

giving the differential equation

$$
-k(T) \frac{d T}{d r}=\frac{1}{3} r H
$$

Integrating from the center of the sphere at radius 0 to radius $R$, with respective temperatures $T_{c}$ and $T_{1}$, this gives

$$
-\int_{T_{c}}^{T_{1}} k(u) d u=\frac{R^{2} H}{6}
$$

For $k(u)=\hat{k}_{0} u^{\beta}$, this becomes

$$
-\frac{\hat{k}_{0}}{1+\beta}\left(T_{1}^{1+\beta}-T_{c}^{1+\beta}\right)=\frac{R^{2} H}{6}
$$

which gives

$$
\left(T_{c}^{1+\beta}-T_{1}^{1+\beta}\right)^{1 /(1+\beta)}=\left[\frac{1+\beta}{\hat{k}_{0} K^{1+\beta}} \frac{R^{2} H}{6}\right]^{1 /(1+\beta)}[\mathrm{K}] .
$$

To give a numerical estimate, for the good thermal insulator Torlon 4203 [13], with density $1.42 \mathrm{~g} \mathrm{~cm}^{-3}$ and with $k(T)=6.13 \times 10^{-3}(T / \mathrm{K})^{2.18} \mathrm{~W} /(\mathrm{m} \mathrm{K})$, so that $\beta=2.18$ and $\hat{k}_{0} \mathrm{~K}^{3.18}=$ $6.13 \times 10^{-3} \mathrm{~W} / \mathrm{m}$, the right hand side of Eq. (16) for $R=50 \mathrm{~cm}$ is $6.1 \mathrm{mK}$.

We have used Torlon as a convenient example for estimates, but probably it would not be the most suitable material for these experiments. As discussed by Pobell [6], amorphous materials are usually rich in two-level systems leading to slow relaxation processes with time-dependent heat release. Moreover, polymers and plastics like Torlon may easily absorb impurities which can also give unreliable thermal properties. For realistic experiments, it would be better to use crystalline insulators (such as sapphire or silicon) or superconducting metals. In both cases the thermal conductivity is much higher than that of Torlon at $T>100 \mathrm{mK}$, but drops as $T^{3}$ and approaches the conductivity of plastic materials like Torlon in the $\mathrm{mK}$ range.

Concerning the feasibility of this experiment, we note that spheres of meter size have already been suspended and cooled down to millikelvin temperature. An example is Minigrail, a 
$\mathrm{CuAl}$ spherical gravitational wave detector, which was suspended through a central rod suspension and cooled in vacuum down to $60 \mathrm{mK}$ within a few days time [14]. In that case, the dominant residual heating mechanism was likely relaxation of defects and hydrogen ortho-para conversion. Both issues can be avoided in a dedicated experiment by a suitable choice of materials.

2. Another geometry with easily solvable heat transfer would use a long cylinder (or parallelepiped, or more generally a rod of uniform cross section) of length $L$ with the "near" end fastened to a heat sink that provides a large enough heat transport rate so that the heat transport from all other surfaces given by Eq. (8) can be ignored. Then the heat transport problem is one dimensional, and the analog of Eq. (13) is the differential equation

$$
k(T) \frac{d T}{d z}=(L-z) H
$$

Again taking $k(u)=\hat{k}_{0} u^{\beta}$ and integrating, the analog of Eq. (16) relating the "far" to the "near" end temperatures at $z=L$ and $z=0$ respectively is

$$
\left(T_{\mathrm{far}}^{1+\beta}-T_{\text {near }}^{1+\beta}\right)^{1 /(1+\beta)}=\left[\frac{1+\beta}{\hat{k}_{0} K^{1+\beta}} \frac{L^{2} H}{2}\right]^{1 /(1+\beta)}[\mathrm{K}] .
$$

For a rod with the parameters quoted above, and $L=50 \mathrm{~cm}$, the right hand side of Eq. (18) is $8.6 \mathrm{mK}$.

In a variant of the rod geometry, one can attach to the "far" end of the rod an object of larger size, which acts as a "CSL noise absorber". The CSL heat released in the absorber can be much larger than that in the rod. The temperature at the "far" end of the rod will then be determined by matching the heat flow per unit area within the rod with the heat flow per unit area $\dot{Q}_{\mathrm{ABS}}$ entering the rod from the absorber, so that Eq. (17) becomes

$$
k(T) \frac{d T}{d z}=(L-z) H+\dot{Q}_{\mathrm{ABS}},
$$

which on integration gives

$$
\left(T_{\mathrm{far}}^{1+\beta}-T_{\text {near }}^{1+\beta}\right)^{1 /(1+\beta)}=\left[\frac{1+\beta}{\hat{k}_{0} K^{1+\beta}}\left(\frac{L^{2} H}{2}+\dot{Q}_{\mathrm{ABS}} L\right)\right]^{1 /(1+\beta)}[\mathrm{K}] .
$$

This provides the freedom of independently tuning the rod thermal conductivity (by making the cross section arbitrarily small) and the heat input from CSL (by choosing the material and size of the CSL absorber). From an experimental point of view this gives a very flexible design. Of course, one must make sure that the heat transport mechanisms of Eq. (8) from 
the surfaces of both the rod and the absorber are kept negligible, as discussed above following Eq. (10).

To conclude, current bounds on the effective noise coupling $\lambda_{\text {eff }}$ for bulk heating of solids show that in designing experiments to test the enhanced rate [2], [3] that makes latent image formation a measurement, it will be important to take the power spectrum of the noise into account. Because thermal transport rates vanish at zero temperature, millikelvin and submillikelvin experiments to further improve the bounds on $\lambda_{\text {eff }}$ by one or two orders of magnitude may be feasible. However, underground operation is probably necessary in order to evade the limiting heating rate from cosmic rays.

\section{ACKNOWLEDGEMENTS}

We wish to thank Angelo Bassi for helpful comments. A.V. acknowledges support from EU FET project TEQ (grant agreement 766900). The authors thank the referee for a careful reading of the paper and helpful comments.

The appendix (except for the addition of the paragraph containing Eq. (A18)) is based on the preprint [15] by one of the authors (SLA). The other author (AV) later noted a preprint by M. Bahrami [16] in which the calculation is done by a non-perturbative method.

\section{APPENDIX A: HEATING THROUGH PHONON EXCITATION IMPLIED BY COLLAPSE MODELS}

In this appendix we calculate the heating rate of a solid through phonons excited by CSL noise with a non-white power spectrum.

\section{Monatomic lattice unit cell}

Consider a system in initial state $i$ with energy $E_{i}=\hbar \omega_{i}$ at time $t=0$, acted on by a perturbation $V$ which at time $t$ leads to a transition to a state $f$ with energy $E_{f}=\hbar \omega_{f}$. Working in the interaction picture, the transition amplitude $c_{f i}(t)$ is given by

$$
\langle f|c(t)| i\rangle \equiv c_{f i}(t)=-\frac{i}{\hbar} \int_{0}^{t} V_{f i}\left(t^{\prime}\right) e^{i \omega_{f i} t^{\prime}} d t^{\prime}
$$


with $\omega_{f i}=\omega_{f}-\omega_{i}$. For $V$ we take the noise coupling in the mass-proportional continuous spontaneous localization (CSL) model,

$$
\begin{aligned}
V & =\int d^{3} z \frac{d W_{t}(\vec{z})}{d t} \mathcal{V}(\vec{z},\{\vec{x}\}), \\
\mathcal{V}(\vec{z},\{\vec{x}\}) & =-\frac{\hbar}{m_{N}} \sum_{\ell} m_{\ell} g\left(\vec{z}-\vec{x}_{\ell}\right),
\end{aligned}
$$

where we have followed the notation used in [17]. Here $\vec{x}_{\ell}$ are the coordinates of atoms of mass $m_{\ell}, g(\vec{x})$ is a spatial correlation function, conventionally taken as a Gaussian

$$
g(\vec{x})=(2 \pi)^{-3 / 2}\left(r_{c}\right)^{-3} e^{-\vec{x}^{2} /\left(2 r_{c}^{2}\right)}=(2 \pi)^{-3} \int d^{3} q e^{-r_{c}^{2} \vec{q}^{2} / 2-i \vec{q} \cdot \vec{x}},
$$

and the non-white noise has expectation $\mathcal{E}$

$$
\mathcal{E}\left[\frac{d W_{t}(\vec{x})}{d t} \frac{d W_{t^{\prime}}(\vec{y})}{d t^{\prime}}\right]=\frac{1}{2 \pi} \int_{-\infty}^{\infty} d \omega \gamma(\omega) e^{-i \omega\left(t-t^{\prime}\right)} \delta^{3}(\vec{x}-\vec{y})
$$

with $\gamma(\omega)=\gamma(-\omega)$ related to the reduction rate parameter $\lambda(\omega)$ by

$$
\gamma(\omega)=8 \pi^{3 / 2} r_{c}^{3} \lambda(\omega)
$$

We wish now to calculate the expectation $\mathcal{E}[E(t)]$ of the energy attained by the system at time $t$, given by

$$
\mathcal{E}[E(t)]=\mathcal{E}\left[\sum_{f} \hbar \omega_{f i}\left|c_{f i}(t)\right|^{2}\right]
$$

Substituting Eqs. (A1) - (A5), carrying out integrations, and using the formulas [18]

$$
\begin{aligned}
\int_{0}^{t} d t^{\prime} e^{i\left(\omega_{f i}-\omega\right) t^{\prime}} & =\frac{e^{i\left(\omega_{f i}-\omega\right) t}-1}{i\left(\omega_{f i}-\omega\right)} \equiv 2 \pi e^{i\left(\omega_{f i}-\omega\right) t / 2} \delta^{(t)}\left(\omega_{f i}-\omega\right), \\
{\left[\delta^{(t)}\left(\omega_{f i}-\omega\right)\right]^{2} } & \simeq \frac{t}{2 \pi} \delta^{(t)}\left(\omega_{f i}-\omega\right),
\end{aligned}
$$

we find in the large $t$ limit the formula for the energy gain rate

$$
t^{-1} \mathcal{E}[E(t)]=\frac{r_{c}^{3}}{\pi^{3 / 2} m_{N}^{2}} \int d^{3} q \sum_{f} e^{-r_{c}^{2} \vec{q}^{2}} \lambda\left(\omega_{f i}\right) \hbar \omega_{f i}\left|\left\langle f\left|\sum_{\ell} m_{\ell} e^{i \vec{q} \cdot \vec{x}_{\ell}}\right| i\right\rangle\right|^{2}
$$

The next step is to evaluate the matrix element appearing in Eq. (A8) by introducing phonon physics, following the exposition in the text of Callaway [19]. We consider first the simplest case of a monatomic lattice with all $m_{\ell}$ equal to $m_{A}$, independent of the index $\ell$, and write the atom coordinate $\vec{x}_{\ell}$ as

$$
\vec{x}_{\ell}=\vec{R}_{\ell}+\vec{u}_{\ell}
$$


with $\vec{R}_{\ell}$ the equilibrium lattice coordinate and with $\vec{u}_{\ell}$ the lattice displacement induced by the noise perturbation. Writing

$$
\sum_{\ell} m_{\ell} e^{i \vec{q} \cdot \vec{x}_{\ell}}=m_{A} \sum_{\ell} e^{i \vec{q} \cdot \vec{R}_{\ell}} e^{i \vec{q} \cdot \vec{u}_{\ell}}
$$

we note that since the Gaussian in Eq. (A8) restricts the magnitude of $\vec{q}$ to be less than of order of $r_{c}^{-1}$, with $r_{c} \sim 10^{-5} \mathrm{~cm}$, whereas the magnitude of the lattice displacement is much smaller than $10^{-8} \mathrm{~cm}$, the exponent in $e^{i \vec{q} \cdot \vec{u}_{\ell}}$ is a very small quantity. So we can Taylor expand to write

$$
e^{i \vec{q} \cdot \vec{u}_{\ell}} \simeq 1+i \vec{q} \cdot \vec{u}_{\ell}
$$

The leading term 1 does not contribute to energy-changing transitions, so we have reduced the matrix element in Eq. (A8) to the simpler form

$$
\left\langle f\left|\sum_{\ell} m_{\ell} e^{i \vec{q} \cdot \vec{x}_{\ell}}\right| i\right\rangle \simeq i m_{A}\left\langle f\left|\sum_{\ell} e^{i \vec{q} \cdot \vec{R}_{\ell}} \vec{q} \cdot \vec{u}_{\ell}\right| i\right\rangle \quad, \quad f \neq i
$$

The approximation leading to Eq. (A12) is a phonon analog of the electric dipole approximation made in electromagnetic radiation rate calculations.

We now substitute the expression [19] for the lattice displacement in terms of phonon creation and annihilation operators,

$$
\vec{u}_{\ell}=\frac{\Omega}{8 \pi^{3}}\left(\frac{\hbar \mathcal{N}}{m_{A}}\right)^{1 / 2} \sum_{j} \int \frac{d^{3} k}{\left(2 \omega_{j}(\vec{k})\right)^{1 / 2}}\left[\vec{e}^{(j)}(\vec{k}) e^{i \vec{k} \cdot \vec{R}_{\ell}} a_{j}(\vec{k})+\vec{e}^{(j) *}(\vec{k}) e^{-i \vec{k} \cdot \vec{R}_{\ell}} a_{j}^{\dagger}(\vec{k})\right],
$$

where the sum on $j$ runs over the acoustic phonon polarization states, and where $\Omega$ and $\mathcal{N}$ are respectively the lattice unit cell volume, and the number of unit cells. Taking the initial state $i$ to be the zero phonon state, only the $a_{j}^{\dagger}$ term in Eq. (A13) contributes, and we can evaluate the sum over lattice sites $\ell$ in Eq. (A12) using the formula [19]

$$
\sum_{\ell} e^{i(\vec{q}-\vec{k}) \cdot \vec{R}_{\ell}}=\frac{8 \pi^{3}}{\Omega} \delta^{3}(\vec{q}-\vec{k})
$$

Carrying out the $\vec{k}$ integration, noting that $\vec{q} \cdot \vec{e}^{(j)}(\vec{q})$ selects the longitudinal phonon with frequency $\omega_{L}(\vec{q})$, defining $\vec{w}=r_{c} \vec{q}$, writing $M=\mathcal{N} m_{A}$ for the total system mass, and assembling all the pieces, we arrive at the answer

$$
\begin{aligned}
t^{-1} \mathcal{E}[E(t)] & =\frac{\hbar^{2} M}{m_{N}^{2} r_{c}^{2}} \frac{1}{2 \pi^{3 / 2}} \int d^{3} w e^{-\vec{w}^{2}} \vec{w}^{2} \lambda\left(\omega_{L}\left(\vec{w} / r_{c}\right)\right)=\frac{3}{4} \frac{\hbar^{2} \lambda_{\text {eff }} M}{m_{N}^{2} r_{c}^{2}}, \\
\lambda_{\text {eff }} & \equiv \frac{2}{3 \pi^{3 / 2}} \int d^{3} w e^{-\vec{w}^{2}} \vec{w}^{2} \lambda\left(\omega_{L}\left(\vec{w} / r_{c}\right)\right) .
\end{aligned}
$$


In the white noise case, where $\lambda(\omega)$ is a constant $\lambda$, we can pull it outside the $\vec{w}$ integral and use

$$
\int d^{3} w e^{-\vec{w}^{2}} \vec{w}^{2}=\frac{3}{2} \pi^{3 / 2}
$$

to get the standard formula [5]

$$
t^{-1} \mathcal{E}[E(t)]=\frac{3}{4} \frac{\hbar^{2} \lambda M}{m_{N}^{2} r_{c}^{2}}
$$

When the noise spectrum has a cutoff below $\omega_{L}(\vec{q})$ for $|\vec{q}| \sim r_{c}^{-1}$, the energy gain rate is sharply reduced. To estimate this, let us assume a Gaussian frequency cutoff of the form $\lambda(\omega)=$ $\lambda \exp \left(-\omega^{2} t_{c}^{2}\right)$, with $t_{c}$ a correlation time, and take $\omega_{L}(\vec{q})=v_{s}|\vec{q}|$, with $v_{s}$ the sound velocity in the solid. Then Eq. (A15) gives

$$
\lambda_{\mathrm{eff}}=\frac{\lambda}{\left(1+v_{s}^{2} t_{c}^{2} / r_{c}^{2}\right)^{5 / 2}} .
$$

Thus, $\lambda \simeq 10^{-7.7} \mathrm{~s}^{-1}$ and $\lambda_{\text {eff }} \leq 10^{-11} \mathrm{~s}^{-1}$ would correspond to $r_{c} / t_{c} \leq v_{s} / 4.5$, strongly ruling out a naive guess $r_{c} / t_{c} \simeq$ light velocity. When Eq. (A18) is approximated as $\lambda_{\text {eff }} \simeq \lambda\left(r_{c} /\left(v_{s} t_{c}\right)\right)^{5}$, it corresponds (up to constant factors) to the estimate given following Eq. (45) of Bahrami [16] which assumes a step function frequency cutoff.

\section{Extensions to multi-atom unit cell and nonzero phonon initial state}

Although we have derived the result of Eq. (A15) for the case of a monatomic lattice and a zero phonon initial state, the result is more general as we shall now show. In the monatomic case, focusing only on the atomic mass factors and longitudinal phonon polarization vectors, Eqs. (A12) and (A13) give a factor

$$
m_{A}^{1 / 2} \vec{e}^{(L) *}(\vec{k}) \simeq m_{A}^{1 / 2} \vec{e}^{(L) *}(\overrightarrow{0})
$$

After the $\simeq \operatorname{sign}$ we have used the fact, noted after Eq. (A10), that the correlation length $r_{C}$ allows only contributions from phonon wavelengths that are long on a lattice scale, corresponding to $\vec{k} \simeq \overrightarrow{0}$. In the multi-atom case, focusing only on acoustic phonons, ${ }^{1}$ the left-hand side of Eq. (A19) is replaced by

$$
m_{\kappa}^{1 / 2} \vec{e}_{\kappa}^{(L) *}(\vec{k})
$$

\footnotetext{
${ }^{1}$ Optical phonons leave the unit cell center of mass stationary, so obey $\sum_{\kappa} m_{\kappa}^{1 / 2} \vec{e}_{\kappa}^{(s)}(\overrightarrow{0})=0$ for any optical phonon mode $s$. Hence for mass-proportional noise coupling, optical phonons do not contribute to the energy gain rate to leading order in $a / r_{C}$, with $a$ the unit cell dimension.
} 
corresponding to Eqs. (1.4.22a,b) of [19], with $\kappa$ labeling an atom in the multi-atom unit cell. Referring now to the unnumbered equation in Callaway [19] between his Eqs. (1.1.22) and (1.1.23), which we write (using the fact that for $\vec{k}=0$ the polarization vectors are real numbers; see Callaway Eq. (1.1.21)) as

$$
m_{\kappa}^{-1 / 2} \vec{e}_{\kappa}^{(L) *}(\overrightarrow{0})=m_{\kappa}^{-1 / 2} \vec{e}_{\kappa}^{(L)}(\overrightarrow{0})=\vec{C}
$$

with $\vec{C}$ a constant, we see that the longitudinal polarization vectors are no longer unit normalized, as in the monatomic case. Instead, the normalization is given in Eq. (1.1.18a) of [19],

$$
\sum_{\kappa} \vec{e}_{\kappa}^{(L) *}(\overrightarrow{0}) \cdot \vec{e}_{\kappa}^{(L)}(\overrightarrow{0})=1
$$

which on substituting Eq. (A21) gives

$$
|\vec{C}|=\left(\sum_{\kappa} m_{\kappa}\right)^{-1 / 2},
$$

and implies for small $\vec{k}$

$$
m_{\kappa}^{1 / 2} \hat{k} \cdot \vec{e}_{\kappa}^{(L) *}(\vec{k}) \simeq m_{\kappa}|\vec{C}|=m_{\kappa}\left(\sum_{\kappa} m_{\kappa}\right)^{-1 / 2} .
$$

Recalling Eqs. (A11)-(A14), summing over $\kappa$ to get the total contribution to the one-phonon creation amplitude, we have

$$
\sum_{\kappa} m_{\kappa}\left(\sum_{\kappa} m_{\kappa}\right)^{-1 / 2}
$$

which when squared gives a factor

$$
\sum_{\kappa} m_{\kappa}=m_{\mathrm{cell}}
$$

which is the total atomic mass in the unit cell. Thus the only change from the monatomic to the multi-atomic case is the replacement of $m_{A}$ by $m_{\text {cell }}$, and since $\mathcal{N} m_{\text {cell }}=M$, the total system mass, the monatomic formula of Eq. (A15) is unchanged. Heuristically, the reason for this is that, as emphasized by Callaway, for $\vec{k}=0$ acoustic phonons Eq. (A21) implies that all "...particles in each unit cell move in parallel with equal amplitudes", and so behave as a single particle with mass $m_{\text {cell }}$.

The above derivation assumes an initial state with no phonons, but this assumption is not needed to get Eq. (A15). When the initial state is constructed from $n$-phonon states, as in a 
thermal ground state, the $a^{\dagger}$ term in Eq. (A13) contributes a term proportional to $(n+1) \omega_{L}$ to the energy gain, while the $a$ term in Eq. (A13) contributes a corresponding term proportional to $-n \omega_{L}$ to the energy gain; the sum of the two terms is proportional to $(n+1-n) \omega_{L}=\omega_{L}$, so $n$ drops out and the formula of Eq. (A15) is recovered. This simplification could have been anticipated from our earlier analysis of the noise-induced energy gain by an oscillator [20], which showed that the rate of energy gain is a constant independent of the number of oscillator quanta that are present.

[1] For reviews of the theory see: A. Bassi and G. C. Ghirardi, Phys. Rep. 379, 257 (2003); P. Pearle, in "Open Systems and Measurements in Relativistic Quantum Field Theory", Lecture Notes in Physics Vol. 526, H.-P. Breuer and F. Petruccione, eds., Springer, Berlin, 1999. For one of the strongest current bounds on the noise coupling strength based on center-of-mass diffusion, coming from the LISA pathfinder mission, see B. Helou, Phys. Rev. D 95, 084054 (2017).

[2] S. L. Adler, J. Phys. A: Math. Theor. 40, 2935, (E) 13501 (2007).

[3] A. Vinante, R. Mezzena, P. Falferi, M Carlesso, and A. Bassi, Phys. Rev. Lett. 119, 110401 (2017).

[4] Alternative recent proposals include: M. Bahrami, M. Paternostro, A. Bassi, and H. Ulbricht, Phys. Rev. Lett. 112, 210404 (2014); S. Nimmrichter, K. Hornberger, and K. Hammerer, Phys. Rev. Lett. 113, 020405 (2014); F. Laloë, W. J. Mullin, and P. Pearle, Phys. Rev. A 90, 052119 (2014); L. Diósi, Phys. Rev. Lett. 114, 050403 (2015); D. Goldwater, M. Paternostro, and P. F. Barker, Phys. Rev. A 94, 010104 (2016).

[5] P. Pearle and E. Squires, Phys. Rev. Lett. 73, 1 (1994), Eq. (2); S. L. Adler, ref [2] op. cit., Eq. (7) and the related comments contained in that paper's references [5] and [6]. For a detailed derivation in the continuous spontaneous localization (CSL) model, see F. Laloë, W. J. Mullin, and P. Pearle, ref [4] op. cit., Appendix A.

[6] F. Pobell, "Matter and Methods at Low Temperatures", Third Edition, Springer (2007). See Sec. 10.5.4 for heating by radioactivity and high energy particles, and Sec. 5.1.2, Eqs. (5.1) and (5.2), for thermal transport of heat to external sources in cryostats.

[7] E. Nararetski, V. O. Kostroun, S. Dimov, R. O. Pohl, and J. M. Parpia, J. Low Temp. Phys. 177, 609 (2004).

[8] I. de Pater and J. J. Lissauer, "Planetary Sciences", Cambridge University Press, Cambridge (2001), pp. 224-5.

[9] A. Bassi, E. Ippoliti, and B. Vacchini, J. Phys. A: Math Gen. 38, 8017 (2005). See A. Smirne and A. Bassi, Sci. Rep. 5, 12518 (2015) for the extension to the dissipative CSL model.

[10] K. Piscicchia, A. Bassi, C. Curceanu, R. Del Grande, S. Donadi, B. C. Hiesmayr, and A. Pichler, arXiv:1710.01973. 
[11] The CUORE collaboration experiment is described in https://cuore.lngs.infn.it/.

[12] D. Rothfuss et al, Philos. Trans. R. Soc. A 374, 20150051 (2016).

[13] G. Ventura et al., Cryogenics 39, 481 (1999). For the density and other properties of torlon: https://www.professionalplastics.com/professionalplastics/content/downloads/Solvay_Torlon_Design_Guide.pdf

[14] A. de Waard et al, Class. Quantum Grav. 23 S79 (2006).

[15] S. L. Adler, "Heating Through Phonon Excitation Implied by Collapse Models", arXiv:1801.00509, v1 and $\mathrm{v} 2$.

[16] M. Bahrami, "Testing Linearity of Quantum Mechanics with a Thermometer", arXiv:1801.03636, v1, $\mathrm{v} 2$, and $\mathrm{v} 3$.

[17] S. L. Adler and F. M. Ramazanoğlu, J. Phys. A: Math. Theor. 40, 13395 (2007), (E) 42, 109801.

[18] C. Cohen-Tannoudji, J. Dupont-Roc, and G. Grynberg, "Atom-Photon Interactions", John Wiley \& Sons, Inc., New York (1992), Eq. (20) on p. 20 and Eq. (49) on p. 35.

[19] J. Callaway, "Quantum Theory of the Solid State, Part A", Academic Press, New York (1974), Chapter 1 and Appendix A. See especially Eq. (1.2.9) on p. 13, Eq. (1.4.22b) on p. 24, and Eq. (A.9b) on p. 354 .

[20] S. L. Adler, J. Phys. A: Math. Gen. 38, 2729 (2005). 